\title{
Assessment of Aortic Atheromatous Plaque and Stiffness by 64-Slice Computed Tomography is Useful for Identifying Patients With Coronary Artery Disease
}

\author{
Takuhiro Okuyama, MD; Shoichi Ehara, MD; Nobuyuki Shirai, MD; Kenichi Sugioka, MD; \\ Hajime Yamashita, MD; Toru Kataoka, MD; Takahiko Naruko, $\mathrm{MD}^{\dagger}$; Toshihide Itoh ${ }^{\ddagger}$; \\ Katharina Otani ${ }^{\ddagger}$; Toshiyuki Matsuoka, MD*; Yuichi Inoue, MD*; Makiko Ueda, MD**; \\ Junichi Yoshikawa, MD ${ }^{\dagger}$; Takeshi Hozumi, MD; Minoru Yoshiyama, MD
}

\begin{abstract}
Background With multislice computed tomography (MSCT) it is possible to visualize the coronary arteries, as well as the aorta, in a single computed tomography scan. Using MSCT, atherosis and sclerosis of the descending thoracic aorta (DTA) were quantified and differences between patients with and without coronary artery disease (CAD) were analyzed.

Methods and Results The population comprised 89 patients who underwent ECG-gated MSCT: 40 patients with suspected CAD by MSCT underwent invasive coronary angiography, and had documented significant stenoses (CAD group), 49 patients did not have significant stenoses (control group). Twenty cross-sectional images of DTA were reconstructed every 5\% (0-95\%) of the RR interval, and the largest and smallest luminal areas were traced. Atheromatous score and stiffness $\beta$ of DTA were quantified; both were significantly higher in the $\mathrm{CAD}$ group than in the controls. Multivariate analysis revealed that the average atheromatous score was an independent factor associated with CAD $(\mathrm{p}<0.005)$.

Conclusion This study demonstrates that atherosis and sclerosis of DTA are associated with CAD. In cases with image quality that is unsatisfactory for interpretation of coronary stenoses, additional assessment of atherosclerosis of the DTA will be useful for identifying patients with CAD. (Circ J 2008; 72: 2021 -2027)
\end{abstract}

Key Words: Atherosclerosis; Computed tomography; Coronary disease; Stiffness

A herosclerosis consists of 2 pathological processes: atherosis characterized by morphologic atheromatous lesions in the intima, and sclerosis characterized by an increase in the stiffness of the vessel walls. Whereas in the past, computed tomography (CT) of the coronary arteries could detect only calcifications, multislice CT (MSCT) can now achieve sufficiently high spatial and temporal resolutions to allow visualization of stenoses ${ }^{1}$ and plaques in the coronary arteries? 2,3

Aortic stiffness has not been readily taken into account for the evaluation of coronary artery disease (CAD), although both in vitro and in vivo studies have shown that MSCT provides a sufficiently accurate determination of aortic luminal dimension, cross-sectional area, and wall thickness to measure the distensibility and stiffness of the aorta 4.5 Aortic stiffness is known to increase with age, but it also correlates with various diseases such as $\mathrm{CAD}^{7}$ and hyper-

(Received April 23, 2008; revised manuscript received July 2, 2008; accepted July 29, 2008; released online October 29, 2008)

Departments of Internal Medicine and Cardiology, *Radiology, **Pathology, Osaka City University Graduate School of Medicine, ${ }^{\dagger}$ Department of Cardiology, Osaka City General Hospital, Osaka Ekisaikai Hospital, Osaka and \$Siemens-Asahi Medical Technologies Ltd, Tokyo, Japan

Mailing address: Shoichi Ehara, MD, Department of Internal Medicine and Cardiology, Osaka City University Graduate School of Medicine, 1-4-3 Asahi-machi, Abeno-ku, Osaka 545-8585, Japan. E-mail: ehara@med.osaka-cu.ac.jp

All rights are reserved to the Japanese Circulation Society. For permissions, please e-mail: cj@j-circ.or.jp tension? or hypercholesterolemia6 So far, atherosis and sclerosis of the descending thoracic aorta (DTA) have been observed and analyzed during transesophageal echocardiography (TEE) performed for indications other than CAD,, 9 Sclerosis could also be estimated by the brachial-ankle pulse wave velocity (baPWV), which has the disadvantage of giving only an average estimation over a large vascular bed.

In contrast, for patients who already have an indication for coronary angiography (CAG) by MSCT, which is noninvasive, it would be a clinically important advantage if the same MSCT data could be used to gain additional information about $\mathrm{CAD}$ through analysis of the aorta at multiple locations. A time-resolved, ECG-gated CT technique to derive aortic distensibility from cyclic cross-sectional area changes has already been validated in a phantom set-up with porcine aortic specimens. Ganten et al reported a negative correlation between abdominal aortic distensibility and aging with the use of this method, albeit using 4- or 16-slice CT5 Distensibility, however, depends on blood pressure, whereas stiffness $\beta$ is considered to be independent of blood pressure!0,11

The diagnostic accuracy of MSCT for detecting coronary artery stenoses has been reported by many investigators, with very high specificity and very high negative predictive values. However, the positive predictive values have been relatively low. Most previous studies did not consist of consecutive patients and most studies excluded images that were unsatisfactory for interpretation. It should also be noted that patients with high heart rates or severe coronary calcification were excluded from the analyses. MSCT scans 


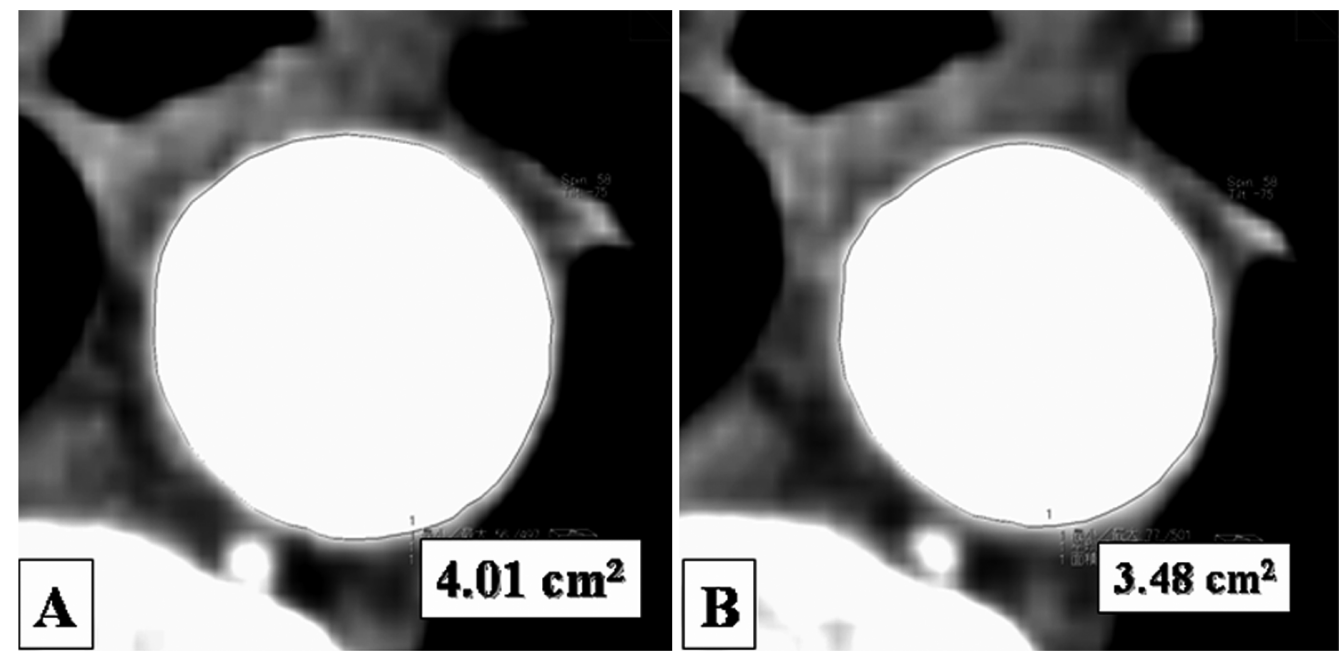

Fig 1. Evaluation of aortic stiffness from ECG-gated time-resolved image series of 20 images per cardiac cycle. (A) Largest luminal area (systolic luminal area) and (B) smallest luminal area (diastolic luminal area), both traced manually on the computer screen.

of the coronary arteries are still associated with a relatively high radiation dose, so it is important to obtain as much relevant information as possible from the scan. The evaluation of atherosclerosis in the aorta, which is often possible even in cases with unsatisfactory coronary artery image quality, could offer a way of increasing the diagnostic usefulness of MSCT for CAD patients.

To the best of our knowledge, a 64-MSCT study documenting the relationship between CAD and atherosclerosis of the DTA has not been reported. The aim of the present study was to evaluate sclerosis and atherosis in the DTA by 64-MSCT at multiple locations and then correlate the results with the occurrence of $\mathrm{CAD}$ in a patient population that comprised those with CAD and those without significant coronary stenoses. The grade of sclerosis was obtained by measuring the aortic stiffness and the grade of atherosis was obtained by evaluating the severity of atheromatous lesions in the DTA at multiple locations.

\section{Methods}

\section{Patients}

The study was based on a retrospective analysis of 89 consecutive patients who had a clinical indication for MSCT angiography for the evaluation of their coronary arteries. All patients underwent contrast-enhanced MSCT in retrospective ECG-gated scanning mode, and invasive CAG. They were divided into 2 groups: the "CAD group" consisted of 40 patients, of whom 28 were suspected of having CAD by MSCT and 12 could not have their coronary arteries assessed because of poor image quality $(n=3)$ or severe calcification $(n=9)$ and who then underwent invasive $C A G$ and were documented to have angiographic narrowing of at least $50 \%$ of the luminal diameter of a major coronary artery; the "control group" consisted of 49 patients, of whom 40 were found not to have significant coronary artery stenoses by MSCT, and underwent confirmatory invasive CAG, but were found not to have significant stenoses, 2 were suspected of having CAD by MSCT and 7 could not be assessed because of poor image quality of their coronary arteries, but who were found to have significant stenoses after undergoing invasive CAG. Patients with atrial fibrilla- tion, frequent extrasystoles, previous coronary artery bypass grafting or percutaneous transluminal intervention were excluded from the study.

The following data were obtained: age, sex, presence of risk factors (smoking defined as having smoked until within the last 3 years, hypertension as defined by the Joint National Committee VI, ${ }^{12}$ diabetes mellitus as defined by the WHO Study Group 13 hypercholesterolemia as defined by the Japan Atherosclerosis Society Guideline ${ }^{14}$ ), body mass index (BMI), blood pressure after image acquisition, heart rates during scanning, and medications before scanning. The BMI was calculated by dividing body weight $(\mathrm{kg})$ by the square of height $(\mathrm{m})$, and BMI $\geq 25.0$ was defined as obesity. The number of diseased vessels was obtained from the invasive CAG examination or from the history of an intervention by counting the number of major coronary arteries with significant stenosis. Serum levels of high-density lipoprotein (HDL)-cholesterol, low-density lipoprotein (LDL)-cholesterol, and triglycerides were also measured in all patients.

The study was approved by the hospital's ethical committee, and informed consent was given by all patients before the study.

\section{MSCT Image Acquisition and Reconstruction}

During a single breath-hold of the patient, image acquisition was performed in the caudal-cranial direction using a 64-slice CT scanner (SOMATOM Sensation 64; Siemens Medical Solutions, Germany). Patients with a heart rate $>65$ beats/min received $20-60 \mathrm{mg}$ metoprolol orally $2 \mathrm{~h}$ before the MSCT scan (CAD group: 35/40 (88\%), control group: $39 / 49(80 \%)$ ). In addition, all patients received $0.6 \mathrm{mg}$ nitroglycerin sublingually immediately before the MSCT scan. The ECG signal was digitally recorded during the scan and all patients included showed sinus rhythm throughout the scan.

First, a non-contrast ECG-gated scan was carried out to determine the calcium score. Then, for the coronary CT angiography, $65-85 \mathrm{ml}$ of contrast medium (Iopamiron 370, Bayer HealthCare, Berlin, Germany), according to the patient's body weight, was injected through a dual-head injector at a rate of $4.0 \mathrm{ml} / \mathrm{s}$ into a cubital vein, followed by 


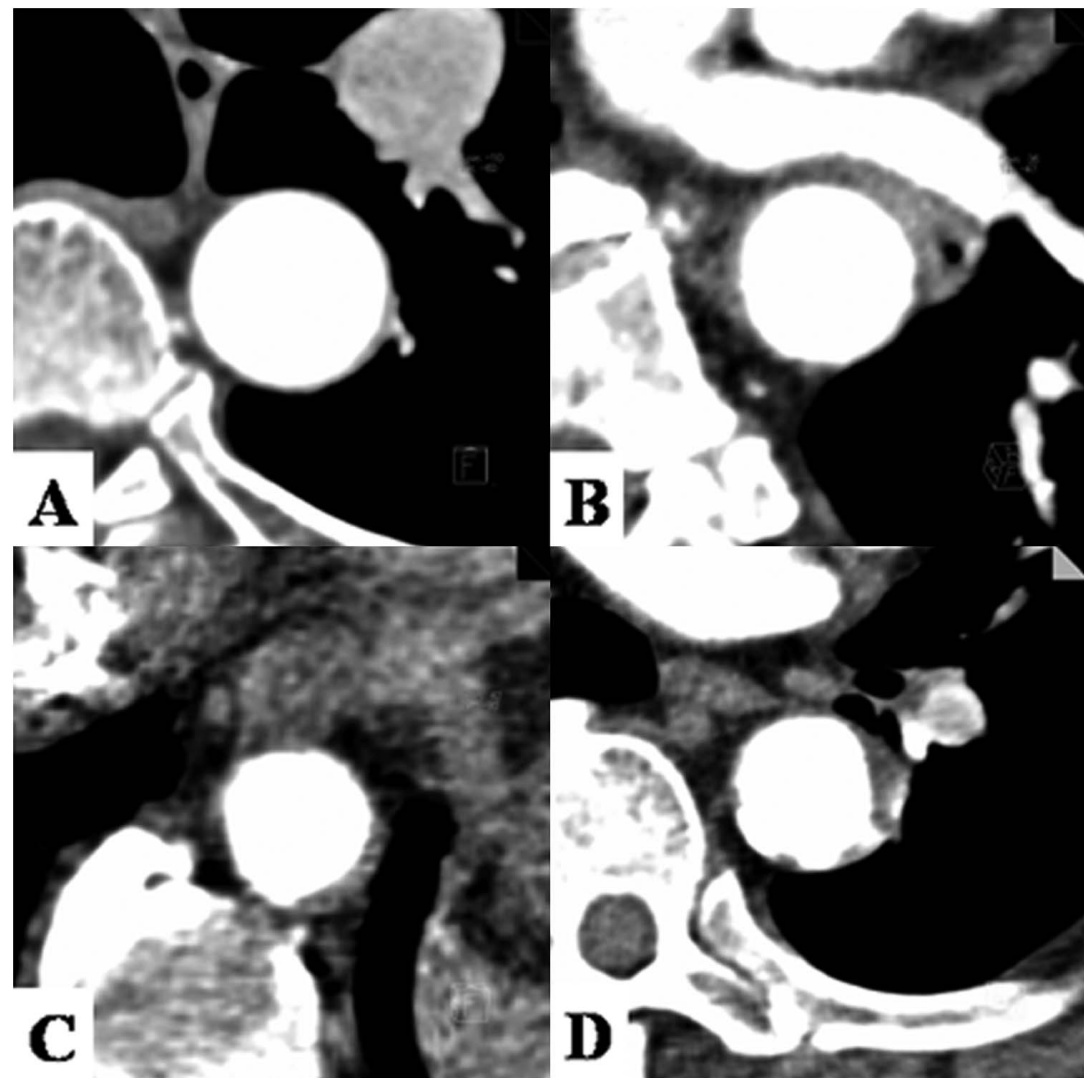

Fig 2. Evaluation of aortic atheromatous score. (A) Score 0 indicates normal aortic wall; (B) score 1 indicates mild atherosis (intimal thickening $<3.0 \mathrm{~mm}$, without intimal irregularities); (C) score 2 indicates moderate atherosis (intimal thickening $\geq 3.0 \mathrm{~mm}$, with intimal irregularities); (D) score 3 indicates severe atherosis (significantly raised plaques, calcified plaques or raised plaques with ulcer formation).
$30 \mathrm{ml}$ of saline solution chaser. The scan delay was determined using a bolus tracking technique. The CT examination was done with a tube voltage of $120 \mathrm{kV}$, an effective tube current-time product of 770 eff mAs, a collimation of $64 \times$ $0.6 \mathrm{~mm}$, a pitch of 0.2 and a gantry rotation time of $330 \mathrm{~ms}$.

Noninvasive brachial arterial blood pressure was registered directly after image acquisition using an arm cuff.

From the raw data of each scan, axial image series were reconstructed every $5 \%(0-95 \%)$ of the RR interval, with an effective slice thickness of $0.75 \mathrm{~mm}$ and an increment of $0.4 \mathrm{~mm}$. A field of view of $180 \times 180 \mathrm{~mm}^{2}$, a $512 \times 512$ matrix and a medium smooth convolution kernel (B25f) were applied.

\section{Evaluation of Sclerosis and Atherosis of the DTA}

First, we evaluated for each patient the stiffness $\beta$ of the DTA as an indicator of aortic sclerosis. ECG-gated timeresolved image series were reconstructed at 20 different phases of the cardiac cycles Cross-sectional images were then selected at 3 levels of the DTA, avoiding sites of severe atheromatous lesions: at the pulmonary artery bifurcation (proximal DTA), below the heart (distal DTA), and in between (middle DTA).

For each level of the DTA, the luminal areas were traced manually on the computer screen. From 20 reconstructed cardiac-phase images, 2 consecutive phases both higher and lower than the end-systolic phase were selected for the measurement of the luminal area. The maximum luminal area was then defined from the largest area of these 5 selected cardiac phases. Similarly, from 20 reconstructed cardiacphase images, 2 consecutive phases both higher and lower than the end-diastolic phase were selected for the measurement of the luminal area. The minimum luminal area was then determined from the smallest area of these 5 selected cardiac phases (Fig 1). The maximum systolic (Dmax) and minimum diastolic (Dmin) lumen diameters were calculated from those areas with the assumption that the cross-section was circular $\left(\text { diameter }=2 \times[\operatorname{area} / \pi]^{1 / 2}\right) !^{15}$ The aortic stiffness $\beta$ was then calculated according to the formula:

$$
\beta=\ln \left(\mathrm{BP}_{\text {sys }} / \mathrm{BP}_{\text {dia }}\right) /\left(\left[\mathrm{D}_{\max }-\mathrm{D}_{\min }\right] / \mathrm{D}_{\min }\right)
$$

where $\ln$ is the natural logarithm, BPsys is the systolic blood pressure, and $\mathrm{BP}_{\mathrm{dia}}$ is the diastolic blood pressure.

The cross-sectional areas were measured by a single experienced observer (TO) who was unaware of the clinical data. To assess interobserver and intraobserver variabilities, cross-sectional areas of 10 randomly selected sites were measured by 2 independent observers and by 1 observer at 2 separate times. Interobserver differences were $0.7 \pm 0.3 \%$ for the systolic luminal area and $0.6 \pm 0.4 \%$ for the diastolic luminal area. Intraobserver differences were $0.6 \pm 0.3 \%$ for the systolic luminal area and $0.5 \pm 0.4 \%$ for the diastolic luminal area.

Next, we assessed the grade of aortic atherosis for each patient. We divided the DTA into 3 segments of $5 \mathrm{~cm}(2.5 \mathrm{~cm}$ proximal and $2.5 \mathrm{~cm}$ distal to each level described above), then continuously assessed the severity of atherosis in the transverse and longitudinal images of each area. We classified the aortic atheromatous lesions into 4 categories and scored each segment according to the severity of atherosis from 0 through 3, as described previously, 16 An atheromatous score 0 indicated a normal aortic wall, score 1 indicated mild atherosis (intimal thickening $<3.0 \mathrm{~mm}$, without intimal irregularities), score 2 indicated moderate atherosis (intimal thickening $\geq 3.0 \mathrm{~mm}$, with intimal irregularities) and score 3 indicated severe atherosis (significantly raised plaques, 
Table 1 Baseline Clinical Characteristics

\begin{tabular}{|c|c|c|c|}
\hline & $C A D(n=40)$ & Control $(n=49)$ & $p$ value \\
\hline Age, years & $67 \pm 9$ & $63 \pm 10$ & 0.08 \\
\hline Male gender, $n$ & $27(68 \%)$ & $29(59 \%)$ & 0.51 \\
\hline Hypertension, $n$ & $27(68 \%)$ & $38(78 \%)$ & 0.34 \\
\hline Hypercholesterolemia, $n$ & $21(53 \%)$ & $23(47 \%)$ & 0.67 \\
\hline Diabetes mellitus, $n$ & $20(50 \%)$ & $5(10 \%)$ & 0.0001 \\
\hline Smoking, $n$ & $22(55 \%)$ & $32(65 \%)$ & 0.39 \\
\hline Obesity $>25 \mathrm{~kg} / \mathrm{m}^{2}, n$ & $9(23 \%)$ & $15(31 \%)$ & 0.47 \\
\hline Multivessel disease, $n$ & $20(50 \%)$ & - & \\
\hline HDL-cholesterol, $\mathrm{mg} / \mathrm{dl}$ & $50 \pm 17$ & $56 \pm 17$ & 0.08 \\
\hline LDL-cholesterol, $\mathrm{mg} / \mathrm{dl}$ & $113 \pm 33$ & $126 \pm 36$ & 0.13 \\
\hline Triglycerides, $\mathrm{mg} / \mathrm{dl}$ & $161 \pm 84$ & $132 \pm 81$ & 0.12 \\
\hline \multicolumn{4}{|l|}{$B P$ after image acquisition } \\
\hline Systole, $\mathrm{mmHg}$ & $145 \pm 22$ & $140 \pm 25$ & 0.30 \\
\hline Diastole, $\mathrm{mmHg}$ & $80 \pm 13$ & $82 \pm 14$ & 0.49 \\
\hline HR during scanning, beats/min & $60 \pm 9$ & $61 \pm 9$ & 0.81 \\
\hline \multicolumn{4}{|l|}{ Medications before scanning } \\
\hline ACE-inhibitors/ARB, $n$ & $16(40 \%)$ & $18(37 \%)$ & 0.83 \\
\hline Calcium antagonists, $n$ & $11(28 \%)$ & $16(33 \%)$ & 0.65 \\
\hline$\beta$-blockers, $n$ & $11(28 \%)$ & $6(12 \%)$ & 0.10 \\
\hline Statins, $n$ & $12(30 \%)$ & $6(12 \%)$ & 0.07 \\
\hline
\end{tabular}

Values are mean $\pm S D$ or $n$ (percentages).

$C A D$, coronary artery disease; $H D L$, high-density lipoprotein; $L D L$, low-density lipoprotein; $B P$, blood pressure; ACE, angiotensinconverting enzyme; $A R B$, angiotensin II type 1 receptor blocker.

Table 2 Atheromatous Scores and Stiffness $\beta$ of the DTA

\begin{tabular}{lccc}
\hline \hline & CAD $(n=40)$ & Control $(n=49)$ & $p$ value \\
\hline Atheromatous score & & & \\
Proximal DTA & $1.50 \pm 0.99$ & $0.63 \pm 0.78$ & $<0.0001$ \\
Middle DTA & $1.68 \pm 1.02$ & $0.69 \pm 0.74$ & $<0.0001$ \\
Distal DTA & $1.83 \pm 0.96$ & $0.76 \pm 0.69$ & $<0.0001$ \\
Maximum & $2.10 \pm 0.90$ & $0.92 \pm 0.81$ & $<0.0001$ \\
Minimum & $1.23 \pm 0.86$ & $0.47 \pm 0.58$ & $<0.0001$ \\
Average & $1.67 \pm 0.82$ & $0.69 \pm 0.66$ & $<0.0001$ \\
Stiffness $\beta$ & & & \\
Proximal DTA & $23.6 \pm 12.4$ & $13.8 \pm 5.6$ & $<0.0001$ \\
Middle DTA & $20.8 \pm 11.3$ & $11.5 \pm 4.7$ & $<0.0001$ \\
Distal DTA & $16.7 \pm 9.0$ & $10.5 \pm 5.6$ & 0.0003 \\
Maximum & $25.3 \pm 12.5$ & $14.7 \pm 5.9$ & $<0.0001$ \\
Minimum & $14.8 \pm 6.8$ & $9.3 \pm 4.2$ & $<0.0001$ \\
Average & $20.3 \pm 9.8$ & $11.9 \pm 4.8$ & $<0.0001$ \\
\hline
\end{tabular}

Values are mean $\pm S D$.

DTA, descending thoracic aorta. See Table 1 for other abbreviation.

calcified plaques or raised plaques with ulcer formation) (Fig 2). In each patient, the maximum and minimum levels of atheromatous score/stiffness, and the average atheromatous score/stiffness were used for the present analysis.

The average stiffness $\beta$ and the average atheromatous score were defined as follows:

average stiffness $\beta=$ (stiffness $\beta$ at proximal DTA + stiffness $\beta$ at middle DTA+stiffness $\beta$ at distal DTA) $/ 3$

average atheromatous $\operatorname{score}=($ atheromatous score at proximal DTA+atheromatous score at middle DTA+ atheromatous score at distal DTA)/3.

\section{Statistical Analysis}

Results are expressed as mean \pm SD. Statistical comparisons of the atheromatous score and the stiffness $\beta$ for the 3 levels of the DTA were performed by 1-way analysis of variance and post-hoc multiple comparison, using Scheffe's test. Categorical variables in Table 1 were compared by chi-square test. The relationship between 2 parameters was evaluated with linear regression analysis. Multivariate logistic regression analysis was performed to identify independent factors associated with CAD. Variables used for analysis were gender, diabetes mellitus, the average atheromatous score, and the average stiffness $\beta$, which associated significantly with CAD in the univariate analyses. Receiveroperating characteristic (ROC) curve analysis was used to determine the cut-off value of the atheromatous score and stiffness $\beta$ to distinguish between patients with and without $\mathrm{CAD}$ at the highest possible sensitivity and specificity levels. Values of $\mathrm{p}<0.05$ were considered significant.

\section{Results}

Patients Characteristics

The clinical characteristics of all 89 patients are shown in Table 1 . There were no statistically significant differences in age, male gender, presence of most risk factors, levels of HDL-cholesterol, LDL-cholesterol, triglycerides, systolic 

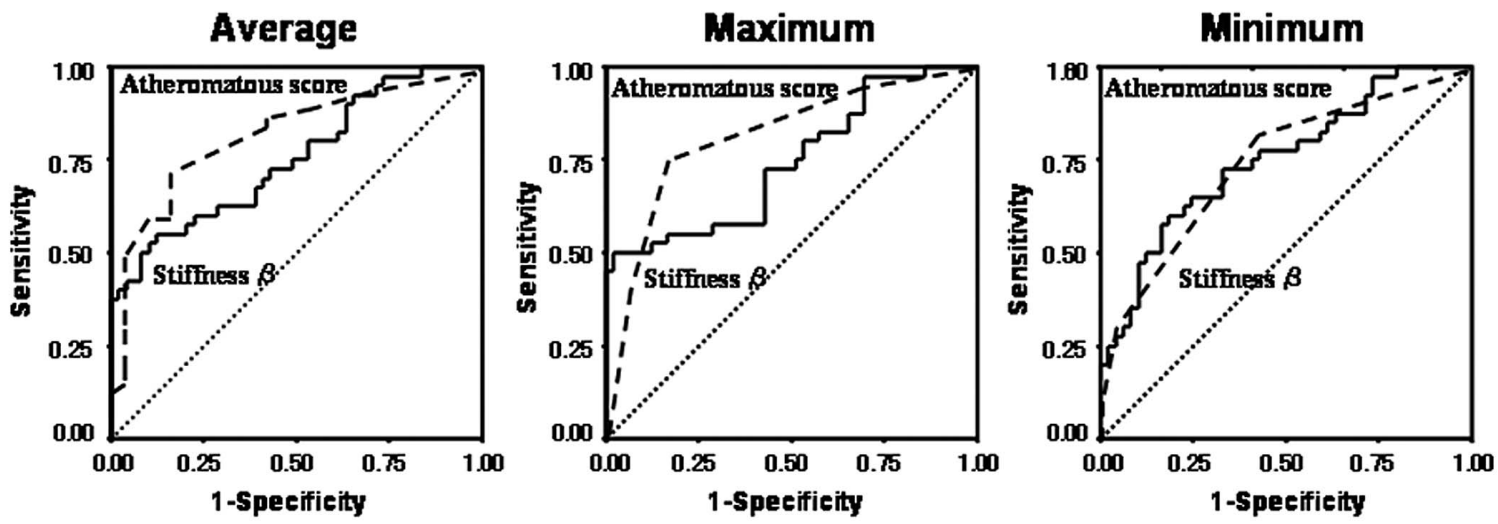

Fig 3. Receiver-operating characteristic curves of the average, maximum, and minimum atheromatous score/stiffness $\beta$ for detection of patients with coronary artery disease. True-positive fraction (sensitivity as y axis) was plotted vs falsepositive fraction (1-specificity as $\mathrm{x}$ axis) by changing cutoff values for test.

or diastolic BP, heart rates or medications before scanning between the CAD and control groups. However, the frequency of diabetes mellitus $(\mathrm{p}<0.0001)$ was significantly higher in the CAD group than in the control group.

\section{Assessment of Aortic Atherosis and Sclerosis}

Table 2 shows the atheromatous score and stiffness $\beta$ for the 2 patient groups; both were significantly higher in the CAD group than in the control group, but no significant differences were found among the atheromatous score of the 3 levels of DTA within either group. However, the stiffness $\beta$ was significantly higher in the proximal DTA than in the distal DTA $(p<0.05)$. Linear regression analysis showed a modest significant correlation between the average atheromatous score and the number of diseased vessels $(r=0.55$; $\mathrm{p}<0.0001$ ), and between the average stiffness $\beta$ and the number of diseased vessels $(\mathrm{r}=0.51 ; \mathrm{p}<0.0001)$.

Furthermore, the average atheromatous score in all patients showed a positive correlation with the average stiffness $\beta(r=0.57, p<0.0001)$. In all patients there were positive correlations between the average atheromatous score and age $(\mathrm{r}=0.36, \mathrm{p}<0.0005)$, and between the average stiffness $\beta$ and age $(\mathrm{r}=0.49, \mathrm{p}<0.0001)$.

\section{Factors Associated With CAD}

To identify the independent factors associated with CAD, multivariate logistic regression analysis was performed using diabetes mellitus, the average atheromatous score, and the average stiffness $\beta$. It revealed that diabetes mellitus (odds ratio $(\mathrm{OR}), 4.667$; 95\% confidence interval (CI), $1.289-16.891 ; \mathrm{p}<0.05)$ and the average atheromatous score (OR 3.462; 95\% CI 1.568-7.644; $\mathrm{p}<0.005$ ) were independent factors associated with CAD. Furthermore, the average atheromatous score was excluded from multivariate analysis because it showed a positive correlation with the average stiffness $\beta$. Diabetes mellitus (OR 5.158; 95\% CI 1.530 17.392; $\mathrm{p}<0.01$ ), and the average stiffness $\beta$ (OR 1.146; 95\% CI 1.057-1.243; $\mathrm{p}<0.001)$ remained as independent factors associated with CAD.

Fig 3 shows the ROC curves of the average, maximum, and minimum atheromatous score and stiffness $\beta$ for identifying patients with CAD. The areas under the curves for the average, maximum, and minimum atheromatous score were 0.82 (95\% CI $0.73-0.91), 0.82$ (95\% CI $0.73-0.91)$ and 0.75 (95\% CI 0.65-0.85), respectively. Regarding the average, maximum, and minimum stiffness $\beta$, the areas under the curves were 0.75 (95\% CI 0.65-0.86), 0.74 (95\% CI $0.64-0.85)$ and 0.75 (95\% CI 0.65-0.85), respectively. A cut-off value $\geq 1.33$ of the average atheromatous score (total atheromatous score $\geq 4$ ) had a sensitivity of $73 \%$, a specificity of $84 \%$, a positive predictive value of $78 \%$, and a negative predictive value of $79 \%$ for detection of CAD. In contrast, for the average stiffness $\beta$, a cut-off value $\geq 14$ had a sensitivity of $63 \%$, a specificity of $65 \%$, a positive predictive value of $60 \%$, and a negative predictive value of $68 \%$. Moreover, the combination of the average atheromatous score $(\geq 1.33)$ and stiffness $\beta(\geq 14)$ had a sensitivity of $48 \%$, a specificity of $92 \%$, a positive predictive value of $83 \%$, and a negative predictive value of $68 \%$ for detecting CAD.

\section{Discussion}

The major finding is that significant differences in the atheromatous score and stiffness $\beta$ of the DTA were found by MSCT of patients with and without CAD. Previous studies have demonstrated that aortic stiffness estimated through baPWV is higher in patients with CAD than in those without CAD!7-19 Arterial stiffness measured by PWV has been identified as an independent cardiovascular risk and has been related to mortality and morbidity $20 \mathrm{Re}$ cently, Fukuda et al demonstrated that baPWV increased with the number of diseased vessels and was significantly correlated with the number of diseased vessels 18 Our present data support those findings. However, baPWV measures PWV between the brachial and ankle arteries, including the aorta and femoral artery, and therefore this method suffers from averaging assessments over a large vascular bed. Moreover, baPWV is unable to identify the atheromatous component at the same time.

In contrast, other noninvasive tools such as MSCT and magnetic resonance imaging enable precise assessment of vascular stiffness and atheromatous lesions in multiple aortic locations in a single image acquisition. As atherosclerosis consists of 2 components, atherosis and sclerosis, studies of atherosclerosis should use a combination or a separation of measures to evaluate both. Currently most diagnostic imaging techniques that detect atherosclerosis focus on the atheromatous component, which is characterized by morphologic changes of the arterial wall. Although the 2 components, atherosis and sclerosis, are interconnected, studies 
examining the association between arterial physiological changes and morphological changes have yielded conflicting results, confirming or denying a close association. It is therefore important to evaluate both components when looking for the mechanisms of progression of atherosclerosis.

Our study also revealed that stiffness is significantly higher in the proximal DTA than in the distal DTA. Rogers et al also showed similar results of PWV assessed by magnetic resonance imaging; that is, PWV in the proximal DTA is significantly higher than PWV in the distal DTA ${ }^{21}$ Structurally, the ratio of elastic fibers to collagen of the aortic wall is regionally heterogeneous, and the ratio changes from 3 in the proximal DTA to 0.8 in the distal DTA22 Rogers et al speculated that progressive fragmentation of elastic fibers and alteration in the regulation of vascular tone may result in a regional increase in PWV primarily affecting the proximal DTA21

TEE can give information about atheromatous lesions and aortic stiffness, but it is usually indicated for clinical cases that do not include all risk factors for atherosclerosis. Typical indications are cardiac embolism, valvular disease and atrial fibrillation, and TEE is not performed for assessing aortic atherosclerosis in patients with CAD. A patient study with the latter aim would pose ethical problems, in particular because TEE causes some discomfort.

So far, only a few studies have reported on the relationship between atherosis and sclerosis evaluated separately by direct measurements in patients with documented CAD, as well as in patients with coronary risk factors, but without significant coronary artery stenoses, as a control group. The capability of MSCT to assess atherosis and sclerosis separately in patients suspected of CAD confirms the clinical significance of this method. As MSCT scans of the coronary arteries are still associated with a relatively high radiation dose, it is important to obtain as much relevant information as possible from 1 scan. Current retrospectively ECG-gated MSCT has been already accepted as an efficient noninvasive tool for the detection of coronary artery stenosis, providing good sensitivity, specificity, and very high negative predictive value, but it must be recognized that these good results have been obtained when technically inadequate scans or patients with rapid heart rates, arrhythmia, or severe calcification were excluded. It should be noted that in the present study no patient was excluded from the assessment of atherosclerosis of the DTA, meaning that the atheromatous score and stiffness $\beta$ of the DTA could be estimated in all patients. Our multivariate analysis demonstrated that the average atheromatous score was an independent factor associated with CAD. A cut-off value $\geq 1.33$ of the average atheromatous score had a sensitivity of $73 \%$, a specificity of $84 \%$, a positive predictive value of $78 \%$, and a negative predictive value of $79 \%$ for detection of CAD, and these values are relatively low. The inclusion of a control group with coronary risk factors may have lead to these results. The combination of the average atheromatous score $(\geq 1.33)$ and stiffness $\beta(\geq 14)$ gave us a higher specificity (92\%) and a higher positive predictive value (83\%). In cases with image quality that is unsatisfactory for interpretation of coronary stenoses, the additional assessment of atherosclerosis of DTA will be useful for identifying patients with CAD.

\section{Study Limitations}

First, our quantitative assessment of atherosclerosis of the DTA is time-consuming. Second, in our study the lumen diameters were calculated from areas where the assumption was that the cross-section was circular, although cross-sectional images avoiding sites of severe atheromatous lesion were selected. Hence, severe atheromatous lesion may cause errors in calculating the lumen diameter. Finally, this was a retrospective study, and we could not evaluate the diagnostic accuracy of MSCT CAG. It will be important to determine in a prospective study whether the average atheromatous score and stiffness of the DTA can identify patients who have CAD, and to assess the added value of analyzing aortic atherosclerosis for the diagnosis of $\mathrm{CAD}$ by MSCT CAG.

\section{Acknowledgments}

The authors gratefully acknowledge the assistance of Atsushi Nakamura, Shunichi Oose, Takuzo Ishikawa, Shinichi Watanabe, Kazuo Okuyama, and other radiological technologists for data acquisition and analysis.

\section{References}

1. Shabestari AA, Abdi S, Akhlaghpoor S, Azadi M, Baharjoo H, Pajouh MD, et al. Diagnostic performance of 64-channel multislice computed tomography in assessment of significant coronary artery disease in symptomatic subjects. Am J Cardiol 2007; 99: 16561661.

2. Motoyama S, Kondo T, Sarai M, Sugiura A, Harigaya H, Sato T, et al. Multislice computed tomographic characteristics of coronary lesions in acute coronary syndromes. J Am Coll Cardiol 2007; 50: 319-326.

3. Jinzaki M, Yamada M, Sato K, Tanami Y, Anzai T, Sasaki K, et al. Overview image of the lumen and vessel wall in coronary CT angiography. Circ J 2008; 72: 671-673.

4. Ganten M, Boese JM, Leitermann D, Semmler W. Quantification of aortic elasticity: Development and experimental validation of a method using computed tomography. Eur Radiol 2005; 15: 25062512.

5. Ganten M, Krautter U, Hosch W, Hansmann J, von Tengg-Kobligk H, Delorme S, et al. Age related changes of human aortic distensibility: Evaluation with ECG-gated CT. Eur Radiol 2007; 17: 701 -708.

6. Tomochika Y, Tanaka N, Ono S, Murata K, Muro A, Yamamura T, et al. Assessment by transesophageal echocardiography of atherosclerosis of the descending thoracic aorta in patients with hypercholesterolemia. Am J Cardiol 1999; 83: 703-709.

7. Herrington DM, Kesler K, Reiber JC, Davis W, Brown WV, Helms $\mathrm{R}$, et al. Arterial compliance adds to conventional risk factors for prediction of angiographic coronary artery disease. Am Heart J 2003; 146: $662-667$

8. Laurent S, Katsahian S, Fassot C, Tropeano AI, Gautier I, Laloux B, et al. Aortic stiffness is an independent predictor of fatal stroke in essential hypertension. Stroke 2003; 34: 1203-1206.

9. Sugioka K, Hozumi T, Sciacca RR, Miyake Y, Titova I, Gaspard G, et al. Impact of aortic stiffness on ischemic stroke in elderly patients. Stroke 2002; 33: 2077-2081.

10. Hayashi K, Handa H, Nagasawa S, Okumura A, Moritake K. Stiffness and elastic behavior of human intracranial and extracranial arteries. $J$ Biomechan 1980; 13: 175-184.

11. Hirai T, Sasayama S, Kawasaki T, Yagi S. Stiffness of systemic arteries in patients with myocardial infarction: A noninvasive method to predict severity of coronary atherosclerosis. Circulation 1989; 80: $78-86$.

12. The sixth report of the Joint National Committee on Prevention, Detection, Evaluation and Treatment of High Blood Pressure. Arch Intern Med 1997; 157: 2413-2446.

13. Report of a WHO Consultation. Definition, diagnosis and classification of diabetes mellitus and its complication. Part 1: Diagnosis and classification of diabetes mellitus. World Health Organization, Department of Noncommunicable Disease Surveillance, Geneva, 1999.

14. Working Committee on JAS Guideline for Diagnosis and Treatment of Hyperlipidemias. Report of the Japan Atherosclerosis Society (JAS) Guideline for Diagnosis and Treatment of Hyperlipidemia in Japanese adults. J Atheroscler Thromb 2002; 9: 1-27.

15. Nakatani S, Yamagishi M, Tamai J, Goto Y, Umeno T, Kawaguchi A, et al. Assessment of coronary artery distensibility by intravascular ultrasound: Application of simultaneous measurements of luminal area and pressure. Circulation 1995; 91: 2904-2910.

16. Davila-Roman VG, Barzilai B, Wareing TH, Murphy SF, Schechtman 
KB, Kouchoukos NT. Atherosclerosis of the ascending aorta: Prevalence and role as an independent predictor of cerebrovascular events in cardiac patients. Stroke 1994; 25: 2010-2016.

17. Park SM, Seo HS, Lim HE, Shin SH, Park CG, Oh DJ, et al. Assessment of arterial stiffness as a clinical parameter for atherosclerotic coronary artery disease. Circ J 2005; 69: 1218-1222.

18. Fukuda D, Yoshiyama M, Shimada K, Yamashita H, Ehara S, Nakamura Y, et al. Relation between aortic stiffness and coronary flow reserve in patients with coronary artery disease. Heart 2006; 92: $759-762$.

19. Koji Y, Tomiyama H, Yamada J, Yambe M, Motobe K, Shiina K, et al. Relationship between arterial stiffness and the risk of coronary artery disease in subjects with and without metabolic syndrome. Hypertens Res 2007; 30: 243-247.

20. Hirata K, Kawakami M, O'Rourke MF. Pulse wave analysis and pulse wave velocity: A review of blood pressure interpretation 100 years after Korotkov. Circ J 2006; 70: 1231-1239.

21. Rogers WJ, Hu YL, Coast D, Vido DA, Kramer CM, Pyeritz RE, et al. Age-associated changes in regional aortic pulse wave velocity. $J$ Am Coll Cardiol 2001; 38: 1123-1129.

22. Apter JT. Correlation of visco-elastic properties with microscopic structure of large arteries. IV: Thermal responses of collagen, elastin, smooth muscle, and intact arteries. Circ Res 1967; 21: 901 -918. 